\title{
Risky Sexual Behavior and Associated Factors Among Sexually-experienced Adolescents in Bangkok, Thailand: Findings From a School Web- based Survey
}

\section{Bang-On Thepthien}

Mahidol University AlHD: Mahidol University ASEAN Institute for Health Development

Celyn Celyn ( $\sim$ celin.mm0501@gmail.com )

Taunggyi University

\section{Research Article}

Keywords: Risky Sexual Behavior, Sexually-experienced, Adolescents

Posted Date: February 15th, 2022

DOI: https://doi.org/10.21203/rs.3.rs-1317940/v1

License: (a) (1) This work is licensed under a Creative Commons Attribution 4.0 International License.

Read Full License 


\section{Abstract}

Background: The risk of sexually transmitted infections (STI) arises when there is unsafe sexual activity. Unsafe sex often begins in the teenage years, and it will persist as long as there is the opportunity for risky sexual activity. The purpose of this study was to assess the sexual risk behaviors and related factors of sexually-active adolescents in educational institutions.

Methods: This was cross-sectional survey using an Internet-based application in schools in Bangkok from November 2020 to February 2021 with a total of 6,167 high school and vocational students. In the study, $14.1 \%$ of all participants had had sexual intercourse before the survey. By gender, $42.1 \%$ of the sexually-active students were males, and $57.9 \%$ were females. Indicators of risky sex include: (1) Not using a condom, (2) Having more than one sex partner; (3) Having sex in exchange for cash or in-kind compensation; and (4) Having sex without consent.

Results: Of a total of 872 sexually-active participants, 590 (67.7\%) had sex risk behavior. The multivariate logistic regression analysis identified the following statistically-significant factors related to risky sex: Alcohol use (AOR $=1.29 ; 95 \% \mathrm{Cl}: 1.03-2.78)$; vaping ( $\mathrm{AOR}=1.59 ; 95 \% \mathrm{Cl}$ : $1.01-2.53)$; cannabis use (AOR $=3.71 ; 95 \% \mathrm{Cl}: 1.51-9.09)$; gambling $(\mathrm{AOR}=1.73 ; 95 \% \mathrm{Cl}: 1.23-2.44)$; unintended pregnancy $(\mathrm{AOR}=$ 2.80; $95 \% \mathrm{Cl}: 1.21-3.57)$; non-use of contraception (AOR $=3.25 ; 95 \% \mathrm{Cl}$ : $2.26-4.68$ ); history of childhood sex abuse $(\mathrm{AOR}=1.67 ; 95 \% \mathrm{Cl}: 1.05-2.68)$.

Conclusions: Our findings suggest that, in designing and implementing sexual risk prevention programs for adolescents in educational settings, there is a need to highlight the role of substance abuse in relation to sexual risk behaviors. Programs should target both male and female students. Teenagers who use marijuana are more likely to be sexually active than those who have never used marijuana. These potential risks should be taken into account with respect to legalization of marijuana and recreational use of this drug.

\section{Plain English Summary}

Risky sexual behavior (RSB) is individuals 'sexual practice that may increase vulnerability of a person to the risk of reproductive health problem. This study was to assess the sexual risk behaviors of students living in Bangkok, which has the highest rates of STIs among regions in Thailand. Previous studies of RSB have focused on the general population age 18 years or older. By contrast, there is a dearth of studies on sex behavior among Thai adolescence age under 18 years. In addition, the present study aimed to explore the association of substance abuse and adverse childhood experiences (ACEs) with adolescent RSB. However, most of those studies were conducted in Western countries; very few have been conducted in Thailand. In this study, $14.1 \%$ of all students in the participating schools had ever had sex before. Using this study criteria, two out of three of the students in this sample had experienced RSB. The findings from this study help inform programs and interventions aimed at reducing the negative health impacts associated with RSB, specifically STIs. The findings of the study relevant for health 
program managers, socio-behavioral researchers, and other stakeholders who design reproductive health intervention strategies for in-school adolescence.

\section{Introduction}

In the world today, there are about 1.2 billion persons between the ages of 10 and 19 years, or $16 \%$ of the total global population. Over half of this age group lives in Asia. South Asia has nearly 350 million persons age 10-19, which is the most of any geographic sub-region. Next most populous is East Asia and the Pacific with over 300 million persons age 10-19 years [1]. Of the 66 million Thais, it is estimated that about seven million are age $10-19$, or $10.7 \%$. Of Bangkok's 5.5 million registered population, about 600,000 are age 10 to 19, or 10.6\% [2]. There is growing concern in Thailand about risky sex behavior (RSB), sexually transmitted infections (STI), and unplanned pregnancy among adolescence. Thus, studies are needed to define the scope of the problem and the factors associated with RSB among adolescents $[3,4]$. The concept of safe sex needs to be promoted as a norm before adolescence become sexually active. Otherwise, it will be more difficult to change RSB once an adolescent acquires unhealthy habits. Adverse consequences of unsafe sex in adolescence include both harmful physical and psychosocial effects [5,6]. In order to prevent the most harmful consequences of unsafe sex (e.g., HIV, incurable STIs, unplanned pregnancy, etc.) it is imperative to promote knowledge about responsible sex behavior before Thai adolescence become sexually active Thus, research into the sexual experience of today's adolescents can help inform the education and prevention programs of tomorrow.

Cultural values and public health policies related to sexual behavior vary from country to country. In addition, cultural values and health policies have changed over time. As a result, gender norms in different parts of the world are experiencing cultural shifts, including Thailand [7]. The risk of the killer virus HIV from unsafe sex has been known around the world for 40 years. Still, there are continuous reports of RSB with each new generation. Today's adolescence have their sexual debut at an increasingly younger age, tend to have sex with multiple casual partners, prefer to use mood-altering drugs before and during sex, and are not conscientious about using effective contraception (especially condoms) [8]. Data for 2020 data indicates that STIs are increasing in many age groups in Thailand, especially among adolescents, with an increase in morbidity from 79 per 100,000 population in 2010 to 191 per 100,000 population ten years later, or more than doubling [9]. This trend is a sign that the reproductive health of today's generation of adolescents is under threat. At present, comprehensive sex education for prepubescent adolescence is woefully inadequate. One of the more serious indicators of this failing is that the leading cause of death for girls aged 15-19 worldwide is complications of pregnancy and childbirth.

Significant evidence suggests that physical abuse and child sexual abuse contribute to RSB in adolescents [10]. Adolescents who were sexually abused in childhood are at higher risk for premature sexual debut, having multiple sex partners, being a victim of coercive sex, engaging in casual sex, and having unprotected sex. Child physical abuse was also associated with a higher risk of sexual behavior at an older age, such as having sex with a casual partner or acquaintance, impulsive sexual behavior, and harmful anal sex in adolescence and young adulthood. Identifying the associations between 
predisposing factors and RSB is essential for the development of effective prevention and intervention strategies to address adolescent reproductive health and prevent negative outcomes, particularly among at-risk populations. According to the literature, there is a link between physical abuse, child sexual abuse, and RSB in adolescence or young adulthood. The mechanisms underlying these connections is unclear. RSB may be used as a means of gaining love and reward after sexual abuse or to alleviate negative emotions caused by childhood abuse. Similarly, risk of substance abuse can increase when teens have difficulty controlling their behavior or when trying to reduce the internal turmoil of adolescence. According to the theory of self-healing, the self-medication theory suggests that a person may abuse substances (e.g., alcohol and other mood-altering drugs) to cope and compensate for the emotional disturbances associated with the harm sustained earlier in life. Substance abuse may lead to an increased risk of addictive sexual behavior by reducing inhibitions. According to myopia theory, alcohol may increase the likelihood of engaging in RSB by limiting perceptions and attention. Because of this, people who are intoxicated and have reduced impulse control, disproportionately focus on the incentives (e.g., arousal, lust) rather than the adverse consequences (e.g., STIs) of sex.

Substance abuse during adolescence interferes with normal cognitive, emotional, and social development. Several studies show that early and frequent substance abuse or increased substance abuse over time threaten development in adulthood, including attainment of educational goals and healthy physical and mental development [11]. A number of studies have examined the relationship between substance abuse and RSB using cross-sectional or longitudinal data covering short periods of time [12]. In general, many of these studies have found a positive association between substance use and RSB. For example, some studies found that excessive alcohol use is positively associated with a greater number of sexual partners, and has a very negative effect on condom use $[12,13,14,15]$. Other studies have found that smoking is strongly associated with higher-risk sexual activity, including having a higher number of sex partners and lack of condom use [16]. The use of marijuana and other moodaltering drugs is associated with having a higher number of sexual partners and lack of condom use [17]. On the other hand, some studies have not found a significant relationship between use of alcohol, marijuana, tobacco, and/or cocaine and condom use $[18,19]$.

In Thailand, secondary school is usually a new environment for the young adolescent, in which people from different primary schools come together and are exposed to a much wider range of attitudes and behaviors of their student peers, including sexual norms and behavior. The vast majority of students attending (general) secondary schools are in their teens, where gender identity formation and gender experimentation take place. Students who are tracked into vocational education usually continue toward a certificate/diploma or commercial college education. By contrast, students in general high schools are exposed to a range of courses and disciplines, usually with the goal of preparing them to pass the college entrance exams for study toward earning a bachelor's degree or higher. Of course, high school and vocational school is a period that coincides with transitioning through puberty, hormonal changes of adolescence, and the experience of powerful drive to explore issues about sex and experiment with sexual behavior [ 20 This is a universal phenomenon of adolescents in all societies around the world - unless the 
state or culture imposes strict restrictions on adolescence attire and unsupervised interaction between the sexes [21].

The objective of this study was to assess the sexual risk behaviors of students living in Bangkok, which has the highest rates of STIs among regions in Thailand. Previous studies of RSB have focused on the general population age 18 years or older. By contrast, there is a dearth of studies on sex behavior among Thai adolescence age under 18 years. In addition, the present study aimed to explore the association of substance abuse and adverse childhood experiences (ACEs) with adolescent RSB. Past research has found a statistically significant association between history of ACEs and RSB in adolescence. However, most of those studies were conducted in Western countries; very few have been conducted in Thailand. The findings from this study should help inform programs and interventions aimed at reducing the negative health impacts associated with RSB, specifically STIs. The findings of the study should be relevant for health program managers, socio-behavioral researchers, and other stakeholders who design reproductive health intervention strategies for in-school adolescence.

\section{Materials And Methods}

\subsection{Study area and period}

Data were collected for this study as part of a Bangkok Behavioral Surveillance Survey (BBSS) project, which monitors HIV infection and risk factors in 10 target populations in the Bangkok metropolitan area. The BBSS is conducted every two years, and includes a sample of students enrolled in high school Years 2 and 5, and vocational Year 2 students from 26 high schools from 15 vocational schools in Bangkok. The cross-sectional data collection was conducted in February 2020 by applying an Internet-based survey using Lime software. The format was optimized for both computers, tablets, and mobile devices. The questionnaire took an average of 15 minutes to complete.

\subsection{Population and eligibility}

The registered population in Bangkok at the time of the survey was 5,588,222 people, with persons age 10-19 years totaling 591,596 people, and persons age 14-16 years totaling 59,139. Data were collected from 6,167 students representing about $10 \%$ of adolescents in educational institutions in Bangkok at the

time. A total of 872 respondents reported that they had ever had sex before, or $14.1 \%$ of all adolescence included in the BBSS. Among the sexually-active students, 66 were studying in high school Year 2, 223 were in high school Year 5, and 583 were in vocational Year 2.

\subsection{Sample size and sampling technique}

The schools were selected by simple random sampling among institutions with at least 1,000 students enrolled, and stratified by six geographic zones of Bangkok. In the final step, 100 -120 students in each school were systematically randomly selected, with an equal number of males and females.

\subsection{Data collection instruments and procedures}


The Sexual Behavior Module was developed from the National Youth Risk Behavior Survey questionnaire. The independent variables used in the study include socio-demographic factors (gender, GPA in the last semester, migration status, location of parents'/guardians' home, other members of the current residence, cost of living, monthly allowance, grade level). Personal behavior variables include alcohol consumption, smoking, vaping, marijuana use, pornography viewing, gambling, ACEs, pregnancy, and contraception. Peer-related factors include having close friends involved in substance abuse, and having close friends who tried to persuade the respondent to use drugs. School-related variables include perception of strict sanctions against student substance abuse and safety. Family factors include whether the parents are divorced and presence of domestic violence. Respondents were asked if they used contraception at last sex. If the response was "yes," they were asked if they used emergency contraception or some other form.

The Substance Abuse Module asked respondents about their use of alcohol, tobacco cigarettes, and ecigarettes (i.e., vaping) in the past month. Respondents were asked about cannabis use in the past year. Alcohol consumption was measured by asking the following question: "During the past month, did you drink one or more servings of an alcoholic beverage at one sitting?" (Beverages included a glass of wine, a bottle of wine cooler, a small bottle/can of beer, a shot of liquor, or a mixed drink.) Smoking (tobacco and or vaping) was measured by the question: "Did you smoke at least once in the past month?" Marijuana use was measured by response to the question: "Did you smoke marijuana at least once in the last year?"

After receiving approval from the Committee, the researchers asked the participating schools to obtain consent from parents of students under 18 years of age to participate in the study. Students were assured that they were free to participate in the survey or not, that their responses would be totally confidential, and their choice to participate or not would have no effect on their student performance evaluation.

\subsection{Measurements and operational definitions}

Risky sexual behavior (RSB) was the primary outcome variable. RSB was measured using a composite of the following indicators: (1) Having sex with a commercial sex worker or sex in exchange for cash or inkind compensation; (2) Having two or more sexual partners; (3-4) Practicing inconsistent condom use or failure to use condoms during intercourse at first and latest sex episode; and (5-6) Experiencing coercion at first sex; experienced coercive sex in the past year. Response was scored, with a total possible score $=$ 6 points. If respondents answered 'Yes' to a particular type of sexual behavior, it was defined as risky sexual behavior. In this study, RSB was calculated only among those students who had had at least one episode of sexual intercourse up to the time of data collection.

\subsection{Data processing and analysis}

The questionnaire was initially checked with the Lime Survey software for completeness and consistency of response prior to the analysis. Data were then exported to SPSS version 25 for further analysis. The researchers conducted descriptive statistics, such as calculating frequency and percentages for discrete independent variables, and the mean (with standard deviation) for continuous variables. A binary logistic 
regression analysis was conducted to test for statistically-significant associations between the independent and dependent variables, with confidence level of $p<0.05$ (95\%), and remaining variables were introduced into the multivariate models. Variables were tested for multi-collinearity using the methodology proposed by Stevens (2012) [22]. The resulting $r$ values were not greater than .80 , and model suitability was examined with the Hosmer-Leme test $(P=0.099)$ with a statistically significant level of $p$-value $<0.05$.

\section{Study Results}

\subsection{Socio-demographic characteristics}

In this study, $14.1 \%(95 \% \mathrm{Cl}: 13.3,15.0)$ of all students in the participating schools had ever had sex before, including $7.6 \%$ of high school Year 2 students, $25.6 \%$ of high school Year 5 students, and $66.9 \%$ of vocational Year 2 students. Of the sexually active, two out of five (42.1\%) were male students, while $57.9 \%$ were female students. Of the 872 students who had ever had sex before, more were female than male, and were more likely to be vocational students, followed by students in high school Years 5 and 2 . Most of the respondents said they were living with their father and/or mother, and were born in Bangkok. About 60 percent lived in the family-owned dwelling. More than half the students perceived that their school was very strict about the prohibition of substance abuse. Half the students had low academic achievement. About one in four lived in a household with divorced parents and/or domestic abuse. Also, about one in four said a close friend tried to persuade them to use addictive drugs, and more male students reported that experience than their female counterparts. 
Table 1

Socio-demographic characteristics of sexually active adolescents in Bangkok, 2020 ( $n=872$ )

\begin{tabular}{|c|c|c|c|c|c|c|c|c|}
\hline \multirow[t]{3}{*}{ Variables } & \multirow[t]{3}{*}{ Categories } & \multirow{2}{*}{\multicolumn{2}{|c|}{$\begin{array}{l}\text { Total }(n= \\
872)\end{array}$}} & \multicolumn{4}{|c|}{ Sex category } & \multirow{3}{*}{$\begin{array}{l}\text { P-Value } \\
\text { a }\end{array}$} \\
\hline & & & & \multicolumn{2}{|c|}{$\begin{array}{l}\text { Male } \\
(n=367)\end{array}$} & \multicolumn{2}{|c|}{$\begin{array}{l}\text { Female } \\
(n=505)\end{array}$} & \\
\hline & & $\mathrm{n}$ & $\%$ & $\mathrm{n}$ & $\%$ & $\mathrm{n}$ & $\%$ & \\
\hline \multirow[t]{2}{*}{ Living arrangements } & $\begin{array}{l}\text { With } \\
\text { biological } \\
\text { parents }\end{array}$ & 610 & 70.0 & 260 & 70.8 & 350 & 69.3 & 0.625 \\
\hline & $\begin{array}{l}\text { Away from } \\
\text { parents }{ }^{b}\end{array}$ & 262 & 30.0 & 107 & 29.2 & 155 & 30.7 & \\
\hline \multirow[t]{3}{*}{ Residence } & Own & 519 & 59.5 & 235 & 64.0 & 284 & 56.2 & $0.006^{*}$ \\
\hline & Rent & 312 & 35.8 & 110 & 30.0 & 202 & 40.0 & \\
\hline & Other & 41 & 4.7 & 22 & 6.0 & 19 & 3.8 & \\
\hline \multirow[t]{2}{*}{ Born in Bangkok } & Yes & 751 & 86.7 & 308 & 83.9 & 448 & 88.7 & $0.040 *$ \\
\hline & No & 166 & 13.3 & 59 & 16.1 & 57 & 11.3 & \\
\hline \multirow[t]{3}{*}{ Current grade level } & $\begin{array}{l}\text { High school } \\
\text { Year } 2\end{array}$ & 66 & 7.6 & 42 & 11.4 & 24 & 4.8 & $0.001^{* *}$ \\
\hline & $\begin{array}{l}\text { High school } \\
\text { Year } 5\end{array}$ & 223 & 25.6 & 96 & 26.2 & 127 & 25.1 & \\
\hline & Vocational 2 & 583 & 66.9 & 229 & 62.4 & 354 & 70.1 & \\
\hline \multirow[t]{2}{*}{ Status } & Live together & 98 & 11.2 & 38 & 10.4 & 60 & 11.9 & 0.481 \\
\hline & No & 774 & 88.8 & 329 & 89.6 & 445 & 88.1 & \\
\hline \multirow[t]{2}{*}{ GPA } & $1-2$ & 475 & 54.5 & 225 & 61.3 & 250 & 49.5 & $0.001^{* *}$ \\
\hline & $3-4$ & 397 & 45.5 & 142 & 38.7 & 255 & 50.5 & \\
\hline \multirow[t]{3}{*}{ Allowance (baht per day) ${ }^{c}$} & $\leq 100$ & 237 & 27.2 & 105 & 28.6 & 132 & 26.1 & 0.64 \\
\hline & $101-200$ & 520 & 59.6 & 204 & 55.6 & 316 & 62.6 & \\
\hline & $>200$ & 115 & 13.2 & 58 & 15.8 & 57 & 11.3 & \\
\hline \multirow{2}{*}{$\begin{array}{l}\text { A close friend tried to } \\
\text { persuade them to use drugs }\end{array}$} & No & 644 & 73.9 & 257 & 70.0 & 387 & 76.6 & $0.028^{*}$ \\
\hline & Yes & 228 & 26.1 & 110 & 30.0 & 118 & 23.4 & \\
\hline
\end{tabular}

Others $^{\mathrm{a}}=\mathrm{Chi}-$ square test, ${ }^{\mathrm{b}}=$ (living with relatives, living alone, living in group) ${ }^{\mathrm{C}}=1 \mathrm{USD}=33$ baht * $=$ p-value $<0.05, * *=p$-value $<0.001$ 


\begin{tabular}{|c|c|c|c|c|c|c|c|c|}
\hline \multirow{2}{*}{$\begin{array}{l}\text { Perception of school } \\
\text { strictness about drugs }\end{array}$} & Low & 410 & 47.0 & 187 & 51.0 & 223 & 44.2 & $0.047 *$ \\
\hline & High & 462 & 53.0 & 180 & 49.0 & 282 & 55.8 & \\
\hline \multirow[t]{2}{*}{ Parents are divorced } & No & 647 & 74.2 & 282 & 76.8 & 365 & 72.3 & 0.129 \\
\hline & Yes & 225 & 25.8 & 85 & 23.2 & 140 & 27.7 & \\
\hline \multirow[t]{2}{*}{ Domestic violence } & No & 670 & 76.8 & 291 & 79.3 & 379 & 75.0 & 0.143 \\
\hline & Yes & 202 & 23.2 & 76 & 20.7 & 126 & 25.0 & \\
\hline \multicolumn{9}{|c|}{ Others $^{a}=$ Chi - square test, ${ }^{b}=$ (living with relatives, living alone, living in group) ${ }^{C}=1$ USD $=33$ baht } \\
\hline \multicolumn{9}{|c|}{$\star=p$-value $<0.05, * *=p$-value $<0.001$} \\
\hline
\end{tabular}

\subsection{Factors related to ACEs and substance abuse}

Over two-thirds of the students (69.2\%) had viewed pornographic media before, with males having a significantly greater proportion of porn-viewing behaviors than females. About one in seven $(13.6 \%)$ of the sample had experienced sexual harassment, while about one in four $(22.6 \%)$ had experienced physical and/or emotional abuse, and physical or emotional neglect/neglect (22.7\%). About one-third have experienced substantial property loss from gambling. Males accounted for significantly more losses from gambling than females. About one in ten had a history of unintended pregnancy; $6.5 \%$ of male students reported that their partner had an unintended pregnancy, while 14.3 percent of female students reported that they had experienced an unintended pregnancy. Use of drugs included drinking at least one alcoholic beverage or a small can/bottle of beer (40.9\%), smoking tobacco $(21.4 \%)$, and smoking ecigarettes $(20.8 \%)$ in the last 30 days. Recreational use of marijuana in the past year was reported by $6.9 \%$, with males having a significantly higher proportion of substance use than females for most substances. The exception is for alcohol consumption, for which females reported significantly higher use than males. Over two-thirds of the respondents (68.8\%) used contraception, and $7.9 \%$ had used emergency contraception. Fully, $7.8 \%$ reported that their first sex was involuntarily, and females had a significantly higher proportion of involuntary sex than males. 
Table 2

Individual related behavior among sexually active adolescents in Bangkok, 2020 ( $\mathrm{n}=872$ )

\begin{tabular}{|c|c|c|c|c|c|c|c|c|}
\hline \multirow[t]{3}{*}{ Variables } & \multirow[t]{3}{*}{ Categories } & \multirow{2}{*}{\multicolumn{2}{|c|}{$\begin{array}{l}\text { Total }(n= \\
872)\end{array}$}} & \multicolumn{4}{|c|}{ Sex category } & \multirow[t]{3}{*}{ P-Value } \\
\hline & & & & \multicolumn{2}{|c|}{$\begin{array}{l}\text { Male } \\
(n=367)\end{array}$} & \multicolumn{2}{|c|}{$\begin{array}{l}\text { Female } \\
(n=505)\end{array}$} & \\
\hline & & $\mathbf{n}$ & $\%$ & $\mathbf{n}$ & $\%$ & $\mathbf{n}$ & $\%$ & \\
\hline \multirow[t]{2}{*}{ Watch porn media } & No & 269 & 30.8 & 53 & 14.4 & 216 & 42.8 & $<0.001$ \\
\hline & Yes & 603 & 69.2 & 314 & 85.6 & 289 & 57.2 & \\
\hline \multirow{2}{*}{$\begin{array}{l}\text { Was physically or emotionally } \\
\text { abused }\end{array}$} & No & 675 & 77.4 & 291 & 79.3 & 384 & 76.0 & 0.257 \\
\hline & Yes & 197 & 22.6 & 76 & 20.7 & 121 & 24.0 & \\
\hline \multirow[t]{2}{*}{ Was sexually abused } & No & 753 & 86.4 & 313 & 85.3 & 440 & 87.1 & 0.434 \\
\hline & Yes & 119 & 13.6 & 54 & 14.7 & 65 & 12.9 & \\
\hline \multirow{2}{*}{$\begin{array}{l}\text { Experienced physical or } \\
\text { emotional neglect }\end{array}$} & No & 674 & 77.3 & 291 & 79.3 & 383 & 75.8 & 0.230 \\
\hline & Yes & 198 & 22.7 & 76 & 20.7 & 122 & 24.2 & \\
\hline \multirow[t]{2}{*}{ Gambles } & No & 587 & 67.3 & 224 & 61.0 & 363 & 71.9 & $0.001 * *$ \\
\hline & Yes & 285 & 32.7 & 143 & 39.0 & 142 & 28.1 & \\
\hline \multirow[t]{2}{*}{ Drinks alcohol } & No & 515 & 59.1 & 236 & 64.3 & 279 & 55.2 & $0.007 *$ \\
\hline & Yes & 357 & 40.9 & 131 & 35.7 & 226 & 44.8 & \\
\hline \multirow[t]{2}{*}{ Smokes cigarettes } & No & 685 & 78.6 & 260 & 70.8 & 425 & 84.2 & $<0.001$ \\
\hline & Yes & 187 & 21.4 & 107 & 29.2 & 80 & 15.8 & \\
\hline \multirow[t]{2}{*}{ Uses cannabis } & No & 812 & 93.1 & 330 & 89.9 & 482 & 95.4 & $0.001 * \star$ \\
\hline & Yes & 60 & 6.9 & 37 & 10.1 & 23 & 4.6 & \\
\hline \multirow[t]{2}{*}{ Use e-cigarettes } & No & 691 & 79.2 & 275 & 74.9 & 416 & 82.4 & $0.007 *$ \\
\hline & Yes & 181 & 20.8 & 92 & 25.1 & 89 & 17.6 & \\
\hline \multirow[t]{2}{*}{ Had an unintended pregnancy } & No & 776 & 89.0 & 343 & 93.5 & 433 & 85.7 & $<0.001$ \\
\hline & Yes & 96 & 11.0 & 24 & 6.5 & 72 & 14.3 & \\
\hline \multirow[t]{2}{*}{ Uses birth control } & No & 272 & 31.2 & 136 & 37.1 & 136 & 26.9 & $0.001 * *$ \\
\hline & Yes & 600 & 68.8 & 231 & 62.9 & 369 & 73.1 & \\
\hline Uses emergency contraception & No & 803 & 92.1 & 347 & 94.6 & 456 & 90.3 & $0.022^{*}$ \\
\hline$*=p$-value $<0.05, * *=$ & 01. & & & & & & & \\
\hline
\end{tabular}




\section{Yes}

69

7.9

20

5.4

49

9.7

$*=p$-value $<0.05, * *=p$-value $<0.001$

\subsection{Risky sexual behavior among study participants}

Risky sex behavior (RSB) was measured across six indicators. As noted above, 7.8\% reported that their first experience of sex was involuntary; $11.4 \%$ had coercive sex in the past year; $33.7 \%$ reported not using a condom at their sexual debut; $30.5 \%$ reported not using a condom the last time they had sex; $46.3 \%$ had more than one sex partner; and 3.0\% had received cash or in-kind compensation for sex in the past year. Using these criteria, two out of three of the students in this sample (67.7\%) had experienced RSB. 
Table 3

Sexual history and family planning use of participants among sexually active adolescents in Bangkok, $2020(n=872)$

\begin{tabular}{|c|c|c|c|c|c|c|c|c|}
\hline \multirow[t]{3}{*}{ Variables } & \multirow[t]{3}{*}{ Categories } & \multirow{2}{*}{\multicolumn{2}{|c|}{$\begin{array}{l}\text { Total }(n= \\
872)\end{array}$}} & \multicolumn{4}{|c|}{ Sex category } & \multirow[t]{3}{*}{ P-Value } \\
\hline & & & & \multicolumn{2}{|c|}{$\begin{array}{l}\text { Male } \\
(n=367)\end{array}$} & \multicolumn{2}{|c|}{$\begin{array}{l}\text { Female } \\
(n=505)\end{array}$} & \\
\hline & & $\mathrm{n}$ & $\%$ & $\mathbf{n}$ & $\%$ & $\mathrm{n}$ & $\%$ & \\
\hline \multirow[t]{2}{*}{ First sex was voluntary } & Yes & 804 & 92.2 & 350 & 95.4 & 545 & 89.9 & $0.003^{*}$ \\
\hline & No & 68 & 7.8 & 17 & 4.6 & 51 & 10.1 & \\
\hline \multirow[t]{2}{*}{ Ever been forced to have sex } & No & 773 & 88.6 & 319 & 86.9 & 454 & 89.9 & 0.171 \\
\hline & Yes & 99 & 11.4 & 48 & 13.1 & 51 & 10.1 & \\
\hline \multirow[t]{2}{*}{ Used condoms at first sex } & Yes & 578 & 66.3 & 231 & 62.9 & 347 & 68.7 & 0.075 \\
\hline & No & 294 & 33.7 & 136 & 37.1 & 158 & 31.3 & \\
\hline \multirow[t]{2}{*}{ Used condom at last sex } & Yes & 606 & 69.5 & 255 & 69.5 & 351 & 69.5 & 0.994 \\
\hline & No & 266 & 30.5 & 112 & 30.5 & 154 & 30.5 & \\
\hline \multirow{2}{*}{$\begin{array}{l}\text { Number of sexual partners in } \\
\text { lifetime }\end{array}$} & One & 468 & 53.7 & 193 & 52.6 & 275 & 54.5 & 0.585 \\
\hline & $\begin{array}{l}\text { More than } \\
\text { one }\end{array}$ & 404 & 46.3 & 174 & 47.4 & 230 & 45.5 & \\
\hline \multirow[t]{2}{*}{ Sold sex before } & No & 846 & 97.0 & 351 & 95.6 & 495 & 98.0 & $0.041 *$ \\
\hline & Yes & 26 & 3.0 & 16 & 4.4 & 10 & 2.0 & \\
\hline \multirow[t]{2}{*}{ Engaged in RSB } & No & 282 & 32.3 & 115 & 31.3 & 167 & 33.1 & 0.589 \\
\hline & Yes & 590 & 67.7 & 252 & 68.7 & 338 & 66.9 & \\
\hline \multirow[t]{2}{*}{ Use birth control } & No & 272 & 31.2 & 136 & 37.1 & 136 & 26.9 & $0.001^{* *}$ \\
\hline & Yes & 600 & 68.8 & 231 & 62.9 & 369 & 73.1 & \\
\hline \multirow{2}{*}{$\begin{array}{l}\text { Use emergency contraception } \\
\text { at last sex }\end{array}$} & No & 803 & 92.1 & 347 & 94.6 & 456 & 90.3 & $0.022^{\star}$ \\
\hline & Yes & 69 & 7.9 & 20 & 5.4 & 49 & 9.7 & \\
\hline \multicolumn{9}{|l|}{ RBS; risky sexual behavior } \\
\hline$*=p$-value $<0.05, * *=p$-value & 001. & & & & & & & \\
\hline
\end{tabular}

\subsection{Results of multivariable logistic regression analysis}


The multivariate logistic regression analysis identified factors that were significantly associated with $\mathrm{RSB}$, as follows: Consuming at least one alcoholic beverage at one sitting (AOR $=1.29 ; 95 \% \mathrm{Cl}$ : $1.03-$ 2.78); smoking e-cigarettes in the past 30 days (AOR $=1.59 ; 95 \% \mathrm{Cl}: 1.01-2.53)$; using marijuana in the past year (AOR $=3.71 ; 95 \% \mathrm{Cl}: 1.51-9.09)$; experiencing substantial loss of property from gambling (AOR $=1.73 ; 95 \% \mathrm{Cl}: 1.23-2.44)$; having had an unintended pregnancy (AOR $=2.80 ; 95 \% \mathrm{Cl}: 1.21-3.57)$; not using contraception (AOR $=3.25 ; 95 \% \mathrm{Cl}$ : $2.26-4.68)$; and/or having a history of ACEs (AOR $=1.67 ; 95 \%$ Cl: $1.05-2.68)$. 
Table 4

Factors associated with risky sexual behavior (RSB) among sexually active adolescents in Bangkok, 2020 $(\mathrm{n}=872)$

\begin{tabular}{|c|c|c|c|c|c|c|c|}
\hline \multirow[t]{2}{*}{ Variables } & \multirow[t]{2}{*}{ Categories } & \multicolumn{2}{|c|}{ RSB n (\%) } & \multirow[t]{2}{*}{ COR } & \multirow[t]{2}{*}{$(95 \% \mathrm{Cl})$} & \multirow[t]{2}{*}{ AOR } & \multirow[t]{2}{*}{$(95 \% \mathrm{Cl})$} \\
\hline & & $\begin{array}{l}\text { No } \\
(n=282)\end{array}$ & $\begin{array}{l}\text { Yes } \\
(n=590)\end{array}$ & & & & \\
\hline \multirow[t]{2}{*}{ Uses cannabis** } & No & $\begin{array}{l}276 \\
(34.0)\end{array}$ & $\begin{array}{l}536 \\
(66.0)\end{array}$ & 1 & & & \\
\hline & Yes & $6(10.0)$ & $54(90.0)$ & 3.67 & $\begin{array}{l}1.50- \\
9.00\end{array}$ & 3.71 & $\begin{array}{l}1.51- \\
9.09\end{array}$ \\
\hline \multirow[t]{2}{*}{ Drinks alcohol* } & No & $\begin{array}{l}186 \\
(36.1)\end{array}$ & $\begin{array}{l}329 \\
(63.9)\end{array}$ & 1 & & & \\
\hline & Yes & $\begin{array}{l}96 \\
(26.9)\end{array}$ & $\begin{array}{l}261 \\
(73.1)\end{array}$ & 1.54 & $\begin{array}{l}1.14- \\
2.07\end{array}$ & 1.29 & $\begin{array}{l}1.03- \\
1.78\end{array}$ \\
\hline \multirow[t]{2}{*}{ Smokes cigarettes } & No & $\begin{array}{l}246 \\
(35.9)\end{array}$ & $\begin{array}{l}439 \\
(64.1)\end{array}$ & 1 & & & \\
\hline & Yes & $\begin{array}{l}36 \\
(19.3)\end{array}$ & $\begin{array}{l}151 \\
(80.7)\end{array}$ & 2.35 & $\begin{array}{l}1.58- \\
3.49\end{array}$ & 1.45 & $\begin{array}{l}0.91- \\
2.31\end{array}$ \\
\hline \multirow[t]{2}{*}{ Uses e-cigarettes* } & No & $\begin{array}{l}246 \\
(35.6)\end{array}$ & $\begin{array}{l}445 \\
(64.4)\end{array}$ & 1 & & & \\
\hline & Yes & $\begin{array}{l}36 \\
(19.9)\end{array}$ & $\begin{array}{l}145 \\
(80.1)\end{array}$ & 2.23 & $\begin{array}{l}1.49- \\
3.31\end{array}$ & 1.59 & $\begin{array}{l}1.01- \\
2.53\end{array}$ \\
\hline \multirow[t]{2}{*}{ Gambles* } & No & $\begin{array}{l}216 \\
(36.8)\end{array}$ & $\begin{array}{l}371 \\
(63.2)\end{array}$ & 1 & & & \\
\hline & Yes & $\begin{array}{l}66 \\
(23.2)\end{array}$ & $\begin{array}{l}219 \\
(76.8)\end{array}$ & 1.93 & $\begin{array}{l}1.40- \\
2.67\end{array}$ & 1.73 & $\begin{array}{l}1.23- \\
2.44\end{array}$ \\
\hline \multirow[t]{2}{*}{$\begin{array}{l}\text { Had an unintended } \\
\text { pregnancy** }\end{array}$} & No & $\begin{array}{l}262 \\
(33.8)\end{array}$ & $\begin{array}{l}514 \\
(66.2)\end{array}$ & 1 & & & \\
\hline & Yes & $\begin{array}{l}20 \\
(20.8)\end{array}$ & $76(79.2)$ & 2.10 & $\begin{array}{l}1.22- \\
3.61\end{array}$ & 2.80 & $\begin{array}{l}1.21- \\
3.57\end{array}$ \\
\hline \multirow[t]{2}{*}{ Uses birth control** } & Yes & $\begin{array}{l}234 \\
(39.0)\end{array}$ & $\begin{array}{l}366 \\
(61.0)\end{array}$ & 1 & & & \\
\hline & No & $\begin{array}{l}48 \\
(17.6)\end{array}$ & $\begin{array}{l}224 \\
(82.4)\end{array}$ & 1.94 & $\begin{array}{l}1.16- \\
3.24\end{array}$ & 3.25 & $\begin{array}{l}2.26- \\
4.68\end{array}$ \\
\hline \multirow[t]{2}{*}{ Was sexually abused* } & No & $\begin{array}{l}254 \\
(33.7)\end{array}$ & $\begin{array}{l}499 \\
(66.3)\end{array}$ & 1 & & & \\
\hline & Yes & $\begin{array}{l}28 \\
(23.5)\end{array}$ & $91(76.5)$ & 1.65 & $\begin{array}{l}1.06- \\
2.59\end{array}$ & 1.67 & $\begin{array}{l}1.05- \\
2.68\end{array}$ \\
\hline
\end{tabular}




\section{Discussion}

In this study, $14.1 \%(95 \% \mathrm{Cl}: 13.3,15.0)$ of all students in the participating schools had ever had sex before. A study of sexual behavior among vocational students in northern Thailand found that $46.5 \% \mathrm{had}$ ever had sex, and males were more sexually active than their female counterparts [7]. This difference with the Bangkok study may be due to varying socio-demographic and cultural context between the two study areas. A study in South Korea [23], found that only $5 \%$ of high school students were sexually active, which is in stark contrast to a study in the Poland where more than $78 \%$ of students attending universities had ever had sex [24]. It is not clear whether these wide differences in sexual activity are attributed to different definitions of sex, time period, or other differences among sample populations and data collection methods.

\subsection{The prevalence of risky sex behavior (RSB)}

Most studies have defined RSB as (1) Not using condoms for every episode of sex; and (2) Having two or more sexual partners. In adolescents, RSB includes sexual debut at a very young age, a factor which is also associated with reproductive health risk, such as unprotected sex. Use of mood-altering drugs before or during sex also contributes to RSB [25]. Some studies define RSB differently as, for example: (1) Number of sexual partners during the lifetime; (2) Inconsistent use of condoms; and (3) Unwanted or unplanned pre-marital pregnancy $[26,27]$. Others also refer to exchanging sex for cash or in-kind compensation as RSB [28]. RSB can produce negative consequences beyond the individual, such as family conflict, damage to relationships, legal disputes, and/or financial problems [29]. Unprotected sex is often the focus as a root cause of these problems because of its association with STIs and unwanted pregnancy. Worldwide, unprotected sex for both male and female adolescents rank second in the health risk category [30]. Over the past 15 years, a large proportion of adolescents in Central and Eastern Europe reported having RSB, which is associated with an increase in STIs other than HIV. The same is true for teen pregnancy and abortion rates [31]. These public health issues have prompted research into the factors influencing adolescent RSB [32]. In addition, definitions of RSB are becoming more standardized, and they now include coercion at sexual debut and forced sex in the past year as criteria. These factors have clinical utility and can be integrated into RSB prevention education programs.

The prevalence of RSB among college and university students in Ethiopia ranged from 23.3-60.9\%. The estimated prevalence of RSB among college and university students was $40.6 \%$ and $42.4 \%$, respectively. The estimated overall prevalence of RSB was $41.6 \%$ [13]. Those results reflect a higher level of RSB than found in other studies conducted in Africa, North America, and Europe. Differences may be due to sample size, age of the sample, calculation of the rates, and definition of RSB [26].

\subsection{Factors associated with RSB}

Adolescent RSB represents a serious public health concern that is detrimental to health and well-being. RSB (e.g., multiple sex partners, not using condoms) negatively affect health by directly contributing to risk for STIs and unintended pregnancy [25]. There is significant evidence suggesting that having a 
history of child sexual abuse contributes to RSB in adolescents [26]. Adolescents who were sexually abused at a young age are at higher risk of premature sex, having sex with multiple partners, having sex under the influence of drugs, having sex with an uncommitted partner, and having unprotected sex [33]. Consistent with this study, adolescents exposed to sexual abuse had 1.67 times more chance of experiencing RSB than adolescents without a history of sexual abuse.

Results reveal a high prevalence of adolescent contraceptive use in Bangkok. More than two-thirds of sexually-active teens in this study have used birth control. Condoms are the most common method of birth control for this adolescence. This is consistent with previous evidence highlighting condoms as the most prevalent method of contraception among adolescents [7,34,]. followed by emergency contraception, and withdrawal [35]. This study did not examine factors which might influence a teen's decision to use contraception. However, the findings of some suggest that the preference for condoms stems from adolescent concern about perceived side effects of other methods [36]. Adolescent girls report using contraception more than their male counterparts. This is different from previous studies in Africa and Asia that found that adolescent males were more likely to use contraception with their female partners [37]. The differences may be attributable to the phrasing of questions about using contraception, cultural differences across countries, and knowledge acquisition through online sources.

The relationship between adolescent sex and substance use has been analyzed. There is evidence from studies that adolescent substance use is predictive of later RSB. Sexually-active teenagers may begin to spend more time with other sexually-active friends, and find themselves in social environments that facilitate substance abuse [11,38]. Adolescent RSB is more common when use of mood-altering substances is involved [39]. A study found that use of addictive drugs before last sex was prevalent among adolescents [38]. This study provides some evidence that is consistent with a possible explanation for the relationship between RSB and adolescent substance use. Substance use disorders are often associated with RSB. For example, studies have shown that, after excessive consumption of alcohol, a person may be more prone to RSB such as promiscuity, neglecting to use a condom, and general reduction of inhibitions which might deter unsafe behavior. Studies have found that RSB often coincides with other deviant behaviors.

The present study analyzed the relationship between alcohol use and RSB among students who had ever had sex. Students who consumed alcohol had more RSB than those who did not consume alcohol. Our findings are consistent with recent studies reporting that alcohol use before sex is more likely to lead to unprotected sex [12-15]. The association between alcohol use and RSB is empirically supported in a variety of studies. However, most of those studies are cross-sectional and, thus, causality cannot be inferred. Some studies have found a significant association between alcohol use and having multiple, concurrent sex partners $[13,39]$. However, there are also inconsistent results from studies of the relationship between alcohol consumption and promiscuity [13] and consistent condom use [40]. Studies have shown that alcohol consumption may lead to RSB by biological mechanisms (i.e., the alcohol myopia theory) in that alcohol inhibits condom use behavior through the pharmacological effect of alcohol on cognitive ability. While alcohol is a well-known behavioral stimulant for sex, mechanisms to 
inhibit RSB (such as prevention of STIs and pregnancy) are inadequate to meet the challenge. The relationship between alcohol use and RSB is complex and may be influenced by a combination of social, physiological, and individualistic personality traits [12]. The relationship between alcohol consumption related to sexual activity and promiscuity may be explained by the alcohol expectancy theory [41]. This theory explains that people who drink alcohol tend to be less nervous, are able to have more sex, and experience a perceived increase in sexual prowess. Teenagers are more likely to consume alcohol before sex when in certain social situations, such as when on group dates, at parties, or at bars [27].

Adolescent cannabis use is a growing public health problem. In this study, the overall prevalence of cannabis use in the past year was approximately $6.9 \%$, and adolescents who used cannabis had 3.7 times more chance of experiencing RSB than adolescents who did not use cannabis. These findings are consistent with previous research which found an association between marijuana use and an increased risk of sex among a sample of adolescents. [42]. Marijuana use is prevalent in high-income countries. For example, in the United States, approximately $0.7 \%$ of $13-14$-year-olds and $3.4 \%$ of $15-16$-year-olds use marijuana daily, and $22.2 \%$ of teens report using marijuana in the past month [43]. The use of marijuana may affect RSB. For example, studies have found that marijuana users are more likely to have their first sex at an earlier age than non-users. Plus, marijuana has been linked to RSB, such as having multiple sex partners. It has been speculated that cannabis use may increase the risk of RSB through negative effects on neurodevelopmental or decision-making abilities [19]. Other factors include personality traits, selfperception, social status, and attractiveness. The main risks from marijuana use include premature sex (e.g., having sex before age 15), promiscuity, and the accompanying risk of STIs, HIV, and unplanned pregnancy $[19,44]$.

The results of this study were not significantly different from previous studies in that $20.8 \%$ of sexuallyexperienced adolescents used e-cigarettes in the last 30 days, and male adolescents engaged in smoking tobacco and e-cigarettes more than their female counterparts. Similarly, research indicates that adolescence e-cigarette use is linked to developmental, physiological, and psychosocial effects, including nicotine addiction, which can cause cardiovascular problems, loss of perception/attention/mood, impulsivity, increased anxiety, and poor academic performance [16]. Additionally, adolescents who used e-cigarettes were more likely to report having four or more sexual partners in their life than non-vapers. The prevalence of condom use at last sex among e-cigarette users was lower than non-e-cigarette users [45]. In the current study, adolescents who smoked e-cigarettes had 1.59 times more chance of having RSB compared to adolescents who did not smoke e-cigarettes. These findings confirm that adolescent substance use is associated with higher rates of involvement in sexual behavior and RSB among adolescents [46Another study found that high school student users of e-cigarettes were more promiscuous than non-users [45]. However, the reason for these relationships is unknown. Studies suggest that perceptions of general behavioral risk are low. High impulsivity and normative perceptions of peer involvement in risky behaviors may lead to multiple substance abuse, RSB, and other health risks [46]. Therefore, these findings highlight the need to further study of the association of e-cigarette use and RSB. A previous study identified intrinsic factors of individuals and normative perceptions of peer 
engagement when trying to develop and implement RSB prevention and health interventions among adolescents.

This study found that one out of three sexually-experienced adolescents had considerable loss of property from gambling. Adolescents who gamble had 1.73 times more chance of RSB compared to adolescents who did not gamble. Male adolescents had more gambling behavior than their female counterparts. This contrasts with some previous studies which found variable prevalence of gambling among adolescents, with $77-88 \%$ of adolescents and young adults generally engaging in one form of gambling or another [47]. That prevalence rate is relatively high compared to the older population, with a prevalence of gambling between $39 \%$ and $50 \%$ [48]. Adolescence are more likely to start social gambling with friends and family, and gambling has the potential to negatively affect an individual's well-being, which may include issues related to relationships, family, finances, social status, and career pursuits [49]. According to a study in Uganda, $62 \%$ of youth gambled, and gamblers were more male than female. The findings from the current study also found that male adolescents gamble more than female adolescents. [50A systematic review of national studies published in 2014 reported that the prevalence of problem gambling in North America was in the range of 2.1-2.6\%, and in Oceania from 0.2-4.4\%. By contrast, in Europe, the prevalence of problem gambling ranged between $0.2 \%$ and $12.3 \%$. These results are hard to verify and may differ across countries and settings due to response bias and survey methodology. Additionally, studies may differ significantly based on inclusion criteria and target populations. These methodological concerns have led to outcomes that vary greatly from country to country or between different studies in the same country [50]. The vast majority of adolescence who gamble consider themselves prone to problem behaviors, and they may not see a link between gambling and health risk, particularly RSB. Gambling is associated with emotional, physical and mental problems, and many adolescences who gamble excessively experience stress, anxiety, and depression. Gambling addicts often experience mood swings that reflect their winnings and losses. However, this uncertainty makes gambling addicts prone to anger and irrational outbursts. These mood disorders also make gamblers more vulnerable to unprotected sex and sex with multiple sex partners, or even exchanging sex for cash or inkind compensation if gambling debt becomes too onerous [50].

Although the study reflects some acceptable quality assurance outcomes, the RSB data in this study were self-reported and, thus, subjects may under- or over-report their own RSB. Also, the survey focused on RSB in the past year, and targeted adolescents in high school Years 2 and 5, and vocational Year 2 students. Thus, the sample is probably not representative of all adolescents, either in Bangkok or Thailand generally. Researchers need to consider studies using community-based qualitative research methods to determine strong predictors of RSB among adolescents. Inquiries about sexual harassment may have inappropriate metrics such as excluding the severity, duration, and frequency of the harassing behavior, and how that affects RSB.

\section{Conclusion}


This study assessed RSB and related factors among sexually-active adolescents enrolled in high school Years 2 and 5, and vocational Year 2, in Bangkok. The students ranged in age from 14-16 years. About $14 \%$ of the study participants had engaged in sexual intercourse in which more than two-third of them involved in the RSB. The findings of this study revealed that substance use variables, such as drinking alcohol, consuming marijuana, and smoking e-cigarettes had a significant association with RSB. Other correlates of RSB include gambling, having had an accidental pregnancy, not used birth control, and had a history of ACEs. This study outlined that the majority of sexually active adolescents are involved in RSB. Interventions at the health facility community and school level should focus on the identified determinants of sexual behaviors among adolescents to minimize the risky consequences.

\section{Abbreviations}

Risky sexual behavior (RSB); Sexually Transmitted Infections (STIs); Human immunodeficiency virus (HIV); acquired immunodeficiency syndrome (AIDS); Adverse Childhood Experiences (ACEs)

\section{Declarations}

\section{Source of funding:}

AIDS, TB and STIs Control Division, Health Department, Bangkok Metropolitan Administration.

\section{Acknowledgements:}

The author thanks students for participated in survey and teachers for their assistance with data collection, all colleague for support and encouragement.

\section{Authors' contributions:}

BT conceptualized the research idea and study design. CC explored the data and performed analysis with the guidelines of BT. BT and CC checked and validated the results. CC drafted the manuscript with the support from BT. BT critically reviewed the manuscript for scientific coherence and supervised the whole study. All authors read and approved the final manuscript.

\section{Data availability:}

The data set gathered and/or analyzed for the current study will be made accessible upon request of the corresponding author.

\section{Ethics approval and consent to participate:}

The project has been approved by the Mahidol University Review Board. (Certificate of approval No: 2019/056). In accordance with the regulations from the MUSSIRB, adolescents aged 18 years and older can make decisions regarding their own health (including participation in health studies), and thus gave 
consent themselves to participate in the current study. Parents/guardians have the right to be informed, and in the current study, all parents/guardians received written information about the study in advance. School attendance were the official reports from the teacher. The student those consented to participate in the study will get password at the day collected data in each school as an appointment in advance by researcher.

\section{Consent for publication:}

Not applicable.

\section{Competing Interests:}

There is no conflict of interest to be reported about this article.

\section{References}

1. Chandra-Mouli V, Ferguson BJ, Plesons M, Paul M, Chalasani S, Amin A, Pallitto C, Sommers M, Avila $\mathrm{R}$, Biaukula KV, Husain S. The political, research, programmatic, and social responses to adolescent sexual and reproductive health and rights in the 25 years since the International Conference on Population and Development. Journal of Adolescent Health. 2019 Dec 1;65(6):S16-40.

2. National Statistical office. The Thai Population Census. (2021). http://www.nso.go.th/sites/2014/Pages/pop.aspx, Accessed 30 Dec 2021.

3. Adeomi AA, Adeoye OA, Adewole A, Israel OA, Temitayo-Oboh A. Sexual risk behaviours among adolescents attending secondary schools in a Southwestern State in Nigeria. Journal of Behavioral Health. 2014;3(3):176-80.

4. Wakasa BF, Oljira L, Demena M, Regassa LD, Daga WB. Risky sexual behavior and associated factors among sexually experienced secondary school students in Guduru, Ethiopia. Preventive Medicine Reports. 2021 Sep 1;23:101398.

5. Tadesse G, Yakob B. Risky sexual behaviors among female youth in Tiss Abay, a semi-urban area of the Amhara Region, Ethiopia. PLoS One. 2015 Mar 4;10(3):e0119050.

6. Irwin CE, Shafer MA. Adolescent sexuality: Negative outcomes of a normative behavior. Adolescents at risk. 2021 Nov 18:35-79.

7. Pinyopornpanish K, Thanamee S, Jiraporncharoen W, Thaikla K, McDonald J, Aramrattana A, Angkurawaranon $\mathrm{C}$. Sexual health, risky sexual behavior and condom use among adolescents' young adults and older adults in Chiang Mai, Thailand: findings from a population-based survey. BMC research notes. 2017 Dec;10(1):1-8.

8. Division of Adolescent and School Health, National Center for HIV/AIDS., Viral Hepatitis, STD, and TB Prevention. Sexual risk behaviors: HIV, STD, \& teen pregnancy prevention. (2021) https://www.cdc.gov/healthyyouth/sexualbehaviors/ Accessed 30 Dec 2021. 
9. Bureau of AIDS, TB and STIs, Ministry of Public Health, STIs epidemiology in Thailand (2021). http://aidssti.ddc.moph.go.th/home. Accessed 30 Nov 2021.

10. Lalor K, McElvaney R. Child sexual abuse, links to later sexual exploitation/high-risk sexual behavior, and prevention/treatment programs. Trauma, Violence, \& Abuse. 2010 Oct;11(4):159-77.

11. Guo J, Chung IJ, Hill KG, Hawkins JD, Catalano RF, Abbott RD. Developmental relationships between adolescent substance use and risky sexual behavior in young adulthood. Journal of adolescent health. 2002 Oct 1;31(4):354-62.

12. Choudhry V, Agardh A, Stafström M, Östergren PO. Patterns of alcohol consumption and risky sexual behavior: a cross-sectional study among Ugandan university students. BMC public health. 2014 Dec;14(1):1-11.

13. Amare T, Yeneabat T, Amare Y. A systematic review and meta-analysis of epidemiology of risky sexual behaviors in college and university students in Ethiopia, 2018. Journal of environmental and public health. 2019 Mar 20;2019.

14. Simbayi LC, Kalichman SC, Jooste S, Mathiti V, Cain D, Cherry C. Alcohol use and sexual risks for HIV infection among men and women receiving sexually transmitted infection clinic services in Cape Town, South Africa. Journal of studies on alcohol. 2004 Jul;65(4):434-42.

15. Fritz KE, Woelk GB, Bassett MT, McFarland WC, Routh JA, Tobaiwa O, Stall RD. The association between alcohol use, sexual risk behavior, and HIV infection among men attending beerhalls in Harare, Zimbabwe. AIDS and Behavior. 2002 Sep;6(3):221-228.

16. Jacobs W, Idoko E, Montgomery L, Smith ML, Merianos AL. Concurrent E-cigarette and marijuana use and health-risk behaviors among US high school students. Preventive Medicine. 2021 Apr $1 ; 145: 106429$.

17. Smith L, Jackson SE, Jacob L, Grabovac I, Nisbet LA, López-Sánchez GF, McDermott D, Salmeron AG, Yang L, Koyanagi A. Association between cannabis use and sexual behavior among adolescents aged 12-15 years in 21 low-and middle-income countries. Addictive behaviors. 2019 Dec 1;99:106073.

18. MacDonald NE, Wells GA, Fisher WA, Warren WK, King MA, Doherty JA, Bowie WR. High-risk STD/HIV behavior among college students. Jama. 1990 Jun 20;263(23):3155- 3159.

19. Lowry R, Holtzman D, Truman BI, Kann L, Collins JL, Kolbe LJ. Substance use and HIV- related sexual behaviors among US high school students: are they related?. American Journal of Public Health. 1994 Jul;84(7):1116-1120.

20. Htike M, Thepthein BO, Chucharoen P. A web-based survey on adverse childhood experience, anxiety, depression, sexual behavior affecting methamphetamine use among adolescents in Bangkok, Thailand. Journal of Public Health and Development. 2017 Nov 30;15(3):17- 31.

21. Srijaiwong S, Sindhu S, Ratinthorn A, Viwatwongkasem C. Factors influencing sexual behaviors among Thai adolescents. Journal of Population and Social Studies [JPSS]. 2017 Jul 1;25(3):171-93.

22. Stevens JP. Applied multivariate statistics for the social sciences. Routledge; 2012 Nov 12. 
23. Valencia ML, Tran BT, Lim MK, Choi KS, Oh JK. Association between socioeconomic status and early initiation of smoking, alcohol drinking, and sexual behavior among Korean adolescents. Asia Pacific Journal of Public Health. 2019 Jul;31(5):443-53.

24. Stokłosa I, Stokłosa M, Porwolik M, Bugajski M, Więckiewicz G, Piegza M, Męcik- Kronenberg T, Gorczyca P. Analysis of High-Risk Sexual Behavior among Polish University Students. International Journal of Environmental Research and Public Health. 2021 Jan;18(7):3737.

25. Koletić G, Kohut T, Štulhofer A. Associations between adolescents' use of sexually explicit material and risky sexual behavior: A longitudinal assessment. PloS one. 2019 Jun 26;14(6):e0218962.

26. Yoon S, Voith LA, Kobulsky JM. Gender differences in pathways from child physical and sexual abuse to adolescent risky sexual behavior among high-risk youth. Journal of Adolescence. $2018 \mathrm{Apr}$ 1;64:89-97.

27. Chawla N, Sarkar S. Defining "high-risk sexual behavior" in the context of substance use. Journal of Psychosexual Health. 2019 Jan;1(1):26-31.

28. Imaledo JA, Peter-Kio OB, Asuquo EO. Pattern of risky sexual behavior and associated factors among undergraduate students of the University of Port Harcourt, Rivers State, Nigeria. Pan African Medical Journal. 2012;12(1).

29. Mirzaei M, Ahmadi K, Saadat SH, Ramezani MA. Instruments of high-risk sexual behavior assessment: A systematic review. Materia socio-medica. 2016 Feb;28(1):46.

30. Stoner SA, George WH, Peters LM, Norris J. Liquid courage: Alcohol fosters risky sexual decisionmaking in individuals with sexual fears. AIDS and Behavior. 2007 Mar;11(2):227-37.

31. Yi H, Shidlo A, Sandfort T. Assessing maladaptive responses to the stress of being at risk of HIV infection among HIV-negative gay men in New York City. Journal of sex research. 2011 Jan 1;48(1):62-73.

32. Maisto SA, Palfai T, Vanable PA, Heath J, Woolf-King SE. The effects of alcohol and sexual arousal on determinants of sexual risk in men who have sex with men. Archives of sexual behavior. 2012 Aug;41(4):971-86.

33. Abajobir AA, Kisely S, Maravilla JC, Williams G, Najman JM. Gender differences in the association between childhood sexual abuse and risky sexual behaviours: A systematic review and metaanalysis. Child abuse \& neglect. 2017 Jan 1;63:249-60.

34. Tangmunkongvorakul A, Carmichael G, Banwell C, Utomo ID, Sleigh A. Sexual perceptions and practices of young people in Northern Thailand. Journal of youth studies. 2011 May 1;14(3):315-39.

35. Yau S, Adamu Y, Wongsawat P, Songthap A. Prevalence and correlates of contraceptive use among vocational schools adolescents in Northern Thailand. Journal of Health Research. 2021 Mar 12. DOI 10.1108/JHR-04-2020-0113

36. Coles MS, Makino KK, Stanwood NL. Contraceptive experiences among adolescents who experience unintended birth. Contraception. 2011 Dec 1;84(6):578-84.

37. Yi S, Te V, Pengpid S, Peltzer K. Social and behavioural factors associated with risky sexual behaviours among university students in nine ASEAN countries: a multi-country cross- sectional 
study. SAHARA-J: Journal of Social Aspects of HIV/AIDS. 2018 Aug 2;15(1):71-9.

38. Clark DA, Donnellan MB, Durbin CE, Nuttall AK, Hicks BM, Robins RW. Sex, drugs, and early emerging risk: Examining the association between sexual debut and substance use across adolescence. PLoS one. 2020 Feb 6;15(2):e0228432.

39. O'Sullivan LF, Thompson AE. Sexuality in adolescence. InAPA handbook of sexuality and psychology, Vol. 1: Person-based approaches. 2014 (pp. 433-486). American Psychological Association.

40. Cooper ML. Alcohol use and risky sexual behavior among college students and youth: evaluating the evidence. Journal of Studies on Alcohol, supplement. 2002 Mar (14):101- 17.

41. Bourne PA, Reid DA, Plummer S, Lewis T, Ferguson J, Foster C, Burke M. The Influence of Alcohol Consumption on Sexual Promiscuity among Young Jamaican Males during the COVID-19 Pandemic. International Journal of Recent Advances in Medical \& Pharma Research. 2020;3(1).

42. El-Menshawi M, Castro G, de la Vega PR, Peláez JG, Barengo NC. First time cannabis use and sexual debut in US high school adolescents. Journal of Adolescent Health. 2019 Feb 1;64(2):194-200.

43. National Institute on Drug Abuse for Teens. Marijuana [Internet]. NIDA for Teens. (2019) cited 2021 Sep 30. Available from: https://teens.drugabuse.gov/drug-facts/marijuana

44. Vasilenko SA, Kugler KC, Rice CE. Timing of first sexual intercourse and young adult health outcomes. Journal of Adolescent Health. 2016 Sep 1;59(3):291-7.

45. Demissie Z, Everett Jones S, Clayton HB, King BA. Adolescent risk behaviors and use of electronic vapor products and cigarettes. Pediatrics. 2017 Feb 1;139(2).

46. Rossi E, Poulin F, Boislard MA. Trajectories of annual number of sexual partners from adolescence to emerging adulthood: individual and family predictors. Journal of youth and adolescence. 2017 May 1;46(5):995-1008.

47. Blinn-Pike L, Worthy SL, Jonkman JN. Adolescent gambling: A review of an emerging field of research. Journal of Adolescent Health. 2010 Sep 1;47(3):223-36.

48. Tse S, Hong SI, Ng KL. Estimating the prevalence of problem gambling among older adults in Singapore. Psychiatry research. 2013 Dec 15;210(2):607-11.

49. MacLaren VV, Fugelsang JA, Harrigan KA, Dixon MJ. The personality of pathological gamblers: A meta-analysis. Clinical psychology review. 2011 Aug 1;31(6):1057-67.

50. Kiwujja V, Mugisha JF. Sexual risk associated with gambling among the youth in Rubaga Division, Kampala. The International journal of health planning and management. 2019 Oct;34(4):1456-68. 